\title{
Взаимосвязь параметров, влияющих на надежность печатных плат
}

\author{
В. Исаев ${ }^{1}$
}

УДК 621.3.049.75:004.3'12 | ВАК 05.11.14

\begin{abstract}
Для предварительного определения надежности печатной платы (ПП), как правило, используют один ключевой критерий: количество переходных отверстий. Результаты такой оценки пригодны лишь для очень ограниченного круга задач, поскольку она не принимает во внимание большое число других параметров, также в большей или меньшей степени влияющих на надежность ПП. Метод, который позволил бы автоматизировать оценку надежности ПП в процессе и по окончании ее проектирования, должен учитывать все значимые параметры и, более того, взаимовлияние различных параметров друг на друга.

В данной статье рассмотрены основные параметры, влияющие на надежность ПП и потому способные служить критериями ее оценки, а также показаны наиболее существенные взаимосвязи между ними.
\end{abstract}

\section{ВВЕДЕНИЕ}

При трассировке сложных плат при помощи САПР обычно остается некоторое количество неразведенных связей, которые конструктору приходится прокладывать вручную. Кроме того, некоторые участки топологии требуют особого подхода, который невозможно или непродуктивно реализовать в виде локальных требований, формализованных в данной САПр; они также требуют вмешательства конструктора.

Решая такие задачи, разработчик ПП неизбежно вносит изменения в тот топологический рисунок, который уже создан программной системой, и эти изменения могут существенно сказаться на общем качестве выполненной трассировки. Разбираться в таких ситуациях помогает опыт, а порой и интуиция специалиста; однако надежнее было бы иметь программный инструмент для быстрой и достоверной оценки влияния любого действия конструктора на работоспособность и надежность проектируемой платы.

С другой стороны, сами САПР не гарантируют достижения максимально возможного качества трассировки, поскольку их алгоритмы построены по принципу достижения наилучшего результата по одному превалирующему параметру. Так, высокопроизводительный автотрассировщик TороR, возможности которого подробно описаны в книге [1], использует превалирующий критерий «Минимальное количество ПО (переходных отверстий)». Вообще же в наиболее популярных современных системах набор приоритетных критериев ограничен практически двумя вариантами: помимо числа

МАИ (НИу), ассистент, isaevtver@gmail.com. переходных отверстий, эту роль играет еще длина связей между компонентами.

Но, кроме двух упомянутых, существуют и другие параметры, пренебрежение которыми может привести к более или менее серьезным недостаткам проводящей структуры, сказывающимся на надежности платы. К ним можно отнести ширину проводников, отношение диаметра отверстия к толщине ПП, класс точности ПП, площадь платы, наличие полигонов, количество слоев ПП и многие другие. Поэтому инструмент, способный оперативно дать оценку произведенной трассировки с учетом всех значащих для надежности параметров топологического рисунка и конструкции платы, найдет применение и для контроля результата работы САПР.

При этом важно понимать, что автономная, изолированная оценка по каждому из критериев недостаточна, поскольку между разнородными параметрами существуют взаимосвязи, и зачастую, улучшая качество трассировки по критерию значения одного из них, можно получить ухудшение по другому критерию.

Задачей данной статьи является анализ основных параметров, влияющих на надежность ПП в той ее части, которая зависит от трассировки: количества переходных отверстий, длины и ширины проводника, класса точности ПП, площади ПП, - и, главное, их взаимозависимости, делающей невозможным достижение наилучших показателей по всем критериям одновременно.

\section{ПЕРЕХОДНЫЕ (МЕЖСЛОЙНЫЕ) ОТВЕРСТИЯ}

Влияние переходных отверстий на надежность ПП обусловлено тем, что переходные отверстия - крайне сложный элемент конструкции ПП, результат 
формирования которого зависит как от качества материалов, так и от соблюдения требований технологических процессов на этапах сверления, прессования, металлизации и травления [2].

Погрешности, привнесенные на этапе сверления, повлекут за собой возникновение целого ряда дефектов на следующих этапах формирования отверстия. Увод сверла может привести к разрыву электрической цепи, если достигнет такой величины, что разрушит связь между гарантийным пояском и соединенным с ним печатным проводником. Затупленное сверло вызывает перегрев материала внутри отверстия; когда будет превышена температура стеклования связующего базового материала ПП, оно, увлекаясь за сверлом, образует на входе и выходе из отверстия буртики эпоксидной смолы, а с ними - поднятие и разрыв фольги гарантийного пояска [3]. Это лишь некоторые примеры критичности корректного сверления для обеспечения работоспособности и надежности ПП.

Не менее важное влияние на получение качественных переходных отверстий оказывает этап прессования. В процессе прессования происходит деформация ПП, что может стать причиной смещения медных площадок под переходные отверстия на внутренних слоях, ведущего к ухудшению характеристик образуемой отверстием электрической цепи вплоть до нарушения ее целостности [4]. ПП со слепыми отверстиями особенно чувствительны к прессованию; неверный подбор режимов может создать температурные и / или механические воздействия, способные привести к разрушению тонких металлизированных стенок отверстий.

Деформацию можно предугадать и скомпенсировать [5], внеся коррективы в проект слоев ПП на этапах конструирования или технологической подготовки производства, однако такая процедура пока еще не получила широкого распространения, поскольку представляет собой сложный математический процесс, учитывающий каждое отверстие - а их на плате могут быть сотни и даже тысячи. Что касается слепых отверстий, то их в проекте ПП можно заменить на сквозные, но это уменьшает полезную площадь платы вплоть до того, что ее трассировка становится невозможной.

Металлизация - самый проблемный из всех технологических процессов. Именно на этом этапе возникает большинство дефектов, ведущих к отказам, связанным с переходными отверстиями. Наиболее распространенные из этих дефектов представлены на рис. 1.

При травлении наиболее уязвимым элементом становится металлизация стенок внутри отверстия. Так, при толщине фольги базового материала, равной 35 мкм, толщина проводников после металлизации составляет 60 мкм, а толщина слоя меди в переходных отверстиях - всего 25 мкм. Попадание раствора травления в отверстия ведет к ухудшению электрических свойств цепи вплоть до нарушения ее целостности и, соответственно, к ослаблению, искажению или полной потере сигнала. Естественно, при более тонкой фольге и малых диаметрах отверстий, характерных
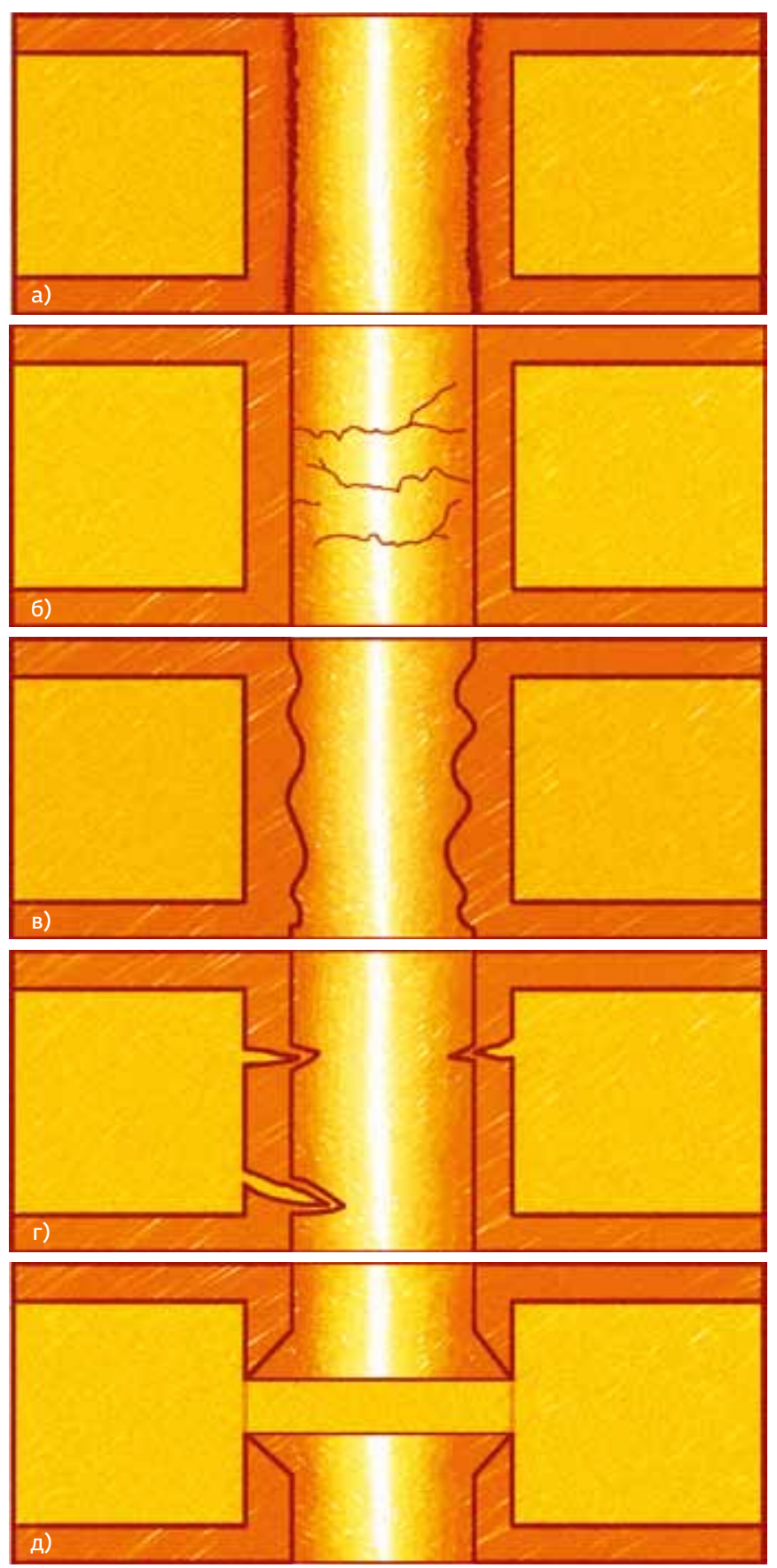

Рис. 1. Дефекты в отверстиях: а - неровность металлизации; б - трещины в металлизации; в - неравномерность металлизации; г - заусенцы; д - отсутствие металлизации 


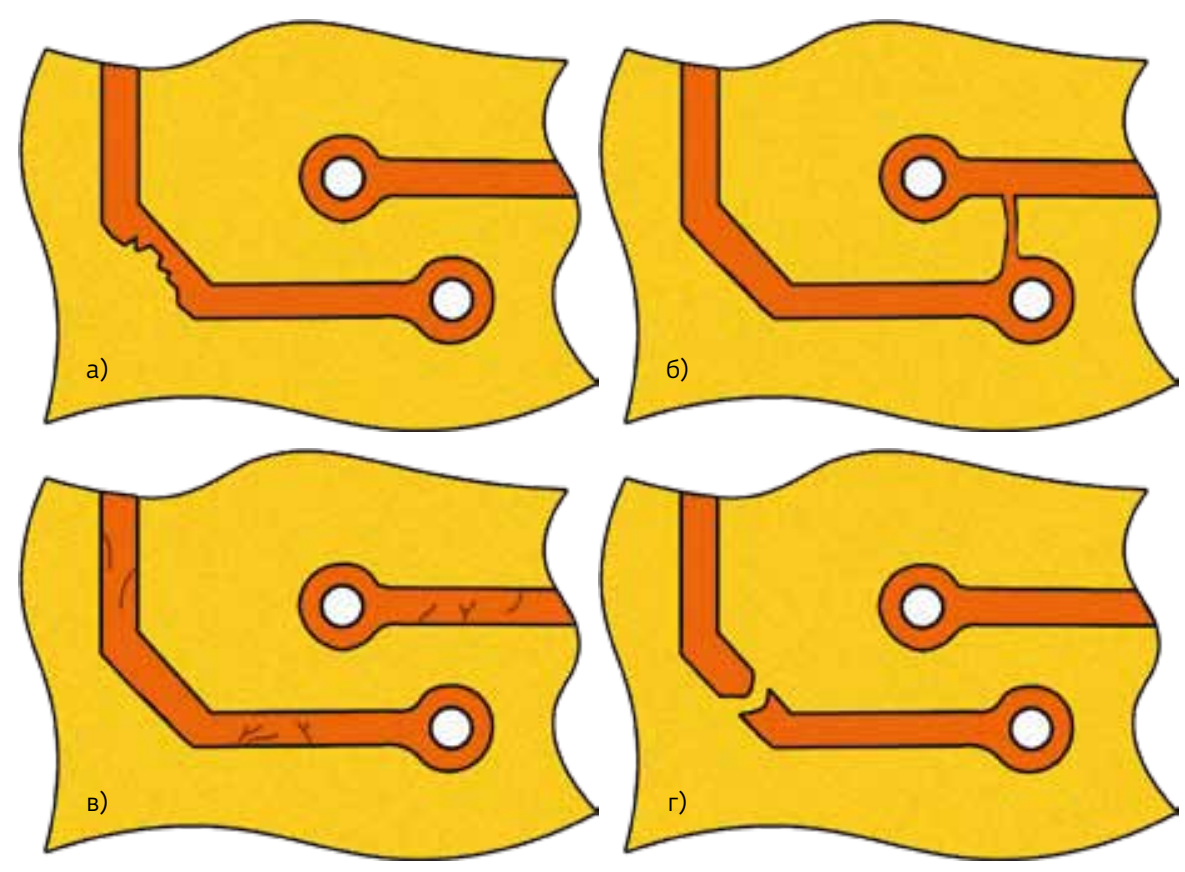

Рис. 2. Дефекты печатных проводников: а - заужение; б - замыкание проводящего рисунка; в - трещины; г - разрыв

для плат высоких классов точности, риск повреждения металлизации отверстия при попадании раствора возрастает. Поэтому, в частности, при использовании тентинг-метода рекомендовано проверять целостность защитного слоя фоторезиста на наличие повреждений в зоне отверстий.

Нужно также упомянуть, что при травлении слоев высвобождаются напряжения базового материала, вызывающие смещение медных площадок под переходные отверстия [6], что также может привести к дефектам внутри отверстий.

\section{ДЛИНА ПЕЧАТНОГО ПРОВОДНИКА}

Важность этого параметра подтверждается тем, что критерий минимальной длины часто принимается в качестве приоритетного в системах автоматической трассировки. Влияние длины проводников на рабочие характеристики и эксплуатационную надежность ПП связано с факторами, которые можно условно разделить на три группы: технологические дефекты, искажение сигналов и эффекты, проявляющиеся на высоких частотах.

К первой группе относятся дефекты, возникающие в ходе изготовления ПП. Такие изъяны, как перетрав или недотрав, микротрещины в материале (рис. 2), являющиеся следствием нарушений технологического процесса или несоответствующего качества исходных материалов, могут появиться в любом месте печатного проводника. Чем он длиннее, тем больше таких локальных дефектов может образоваться на нем, и их суммарное воздействие на электрические характеристики проводника может стать критическим для надежной работы ПП.

С точки зрения влияния на качество сигнала большая длина проводника ведет к росту его сопротивления и, следовательно, к затуханию проходящего по нему сигнала. Также не стоит забывать об изгибах. Длинный проводник крайне редко бывает прямым, и зачастую чем он длиннее, тем больше элементов огибает, образуя большое число изгибов. В месте изгиба ширина проводника изменяется; величина изменения зависит от угла, на который трасса меняет направление (рис. 3), но в любом случае в этом месте резко уменьшается сопротивление - в 1,23 раза для угла $45^{\circ}$ и почти в два раза для угла 90. Уменьшение сопротивления при неизменном напряжении вызывает скачок тока, что ведет к возникновению помехи в проходящем по проводнику сигнале. Поскольку скачок происходит при каждом повороте, то понятно, что на длинном проводнике с множеством изгибов "загрязнение» сигнала может быть весьма значительным.

Что касается высоких частот, то возникающие между длинными проводниками паразитные емкости и индуктивности существенно влияют на качество сигнала, в первую очередь на скорость его нарастания и спада, что для аналогового сигнала означает искажение его формы на пути между источником и приемником, а для цифрового способно привести к нарушениюего целостности. Также стоит упомянуть и скин-эффект.

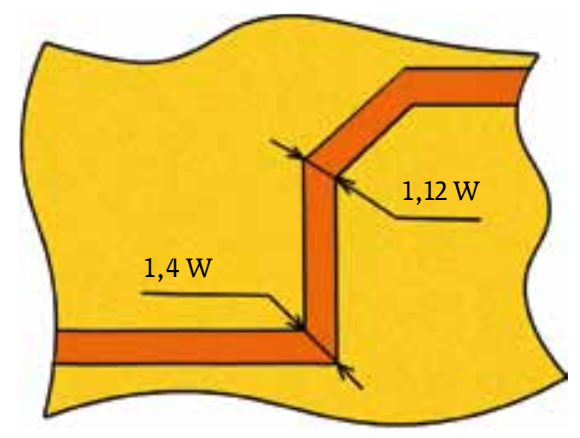

Рис. 3. Изменение ширины проводника в зависимости от угла изгиба 
МЕЖАУНАРОАНАЯ ВЫСТАВКА

$x \rightarrow$
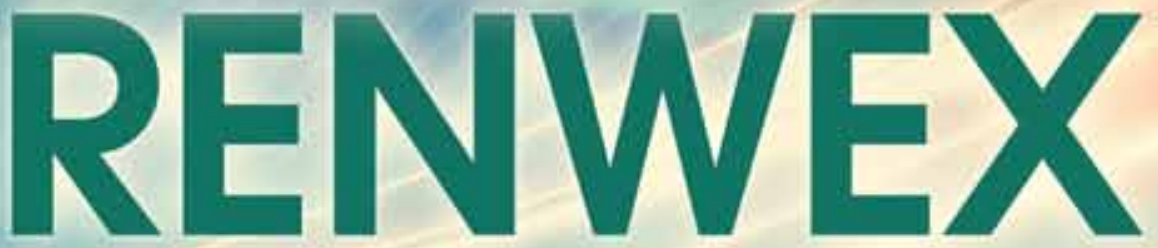

“Возобновляемая энергетика и электротранспорт"

МеЖАУНарОАНЫЙ ФОрУМ “ВозобновАяемая энергетика А^я регионального развития"

\section{0-22 ОКТЯБРЯ 2020}

Россия, Москва, ЦВК "ЭКСПОЦЕНТР", павильон №3

КЛЮЧЕВЫЕ НАПРАВЛЕНИЯ:

(88. Развитие розничного рынка ВИз

и необходимых технических решений

(E) Нормативное регулирование ВИэ

(2) Использование ВИЭ для энергоснабжения удаленных и изолированных потребителей

(9) Использование биотоплива и утилизация отходов

(8) Международный опыт развития возобновляемой энергетики

(2) Цифровизация современной энергетики

Р. Развитие систем накопления энергии для промышленных потребителей и домохозяйств

\$2 Развитие электротранспорта и сопутствующей инфраструктуры

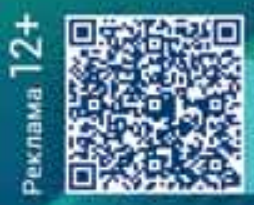

\section{www.renwex.ru}

89

A минпромторг euro

eyro
solde

:

A

B 셰드를

IOA Datpointom 
Вытеснение сигнала в тонкий приповерхностный слой проводника при большой длине последнего грозит заметным затуханием. Еще серьезнее могут быть последствия шероховатости поверхности меди, значительно увеличивающей расстояние, которое проходит сигнал в приповерхностном слое. В результате приходится иметь дело не только с возросшим затуханием сигнала, но и с риском рассинхронизации параллельных шин, ведь шероховатость поверхности описывается только допуском и может существенно различаться у разных проводников.

Конструкторы всегда пытаются минимизировать длину связей, даже тогда, когда более высокий приоритет имеет другой критерий, например количество переходных отверстий. Возникает конкуренция критериев, и конструктору приходится в каждом конкретном случае принимать самостоятельное решение.

Рассмотрим классический пример с подключением двух микросхем. Если их выводы соединяются так, как показано на рис. 4а, то для минимизации связи достаточно поднять микросхему на один шаг сетки вверх (рис. 4б). Если же связи пересекаются (рис. 5а), то ситуация усложняется. С одной стороны, можно провести связи минимальной длины, но добавить два переходных отверстия (рис. 5б), с другой - можно не устраивать дополнительные переходные отверстия, но тогда придется удлинить одну из связей (рис. 5в).

В данном примере с точки зрения обеспечения надежности, видимо, следует принять вариант, представленный на рис. 5в, - ведь удлинение связи незначительно, и очевидно, что оно меньше повлияет на надежность, нежели два переходных отверстия. Но надо понимать, что в примере рассмотрены две микросхемы, стоящие рядом, и, главное, - только две связи. В реальных схемах таких коллизий десятки и сотни, и дилемма - длина связи или дополнительные отверстия? - возникает постоянно.

Для целей данной статьи важно отметить, что рассматривать длину связи в отрыве от других параметров некорректно - так же, как некорректно абсолютизировать задачу уменьшения числа переходных отверстий, обсужденных выше, вне увязки с ее влиянием на остальные критерии надежности

\section{ШИРИНА ПРОВОДНИКА}

Ширина печатного проводника - один из немногих параметров, увеличение значения которого положительно сказывается на надежности и электрических характеристиках печатных плат. Чем шире проводник, тем меньше его сопротивление, меньше потери сигнала, больше токонесущая способность [7]. Путем увеличения ширины проводника можно легко скомпенсировать многие недостатки длинных связей, что еще раз подтверждает взаимосвязь параметров, влияющих на надежность ПП. Однако увеличение этого параметра, с одной стороны, не всегда возможно, а с другой - влечет не только положительные, но и отрицательные последствия.

Первый и очевидный момент: чем шире проводники, тем меньшая номенклатура компонентов может быть использована. Например, подавляющее большинство современных программируемых микросхем, монтируемых на поверхность печатных плат, выпускается в корпусах с шагом контактов не более 0,25 мм, что требует применения пп 3-го и выше класса точности. При наличии в составе устройства таких микросхем разработчик должен установить минимальную ширину проводника, которая позволит подключиться к их контактам; при этом на участках за пределами

Рис. 4. Пример с неперекрестными связями
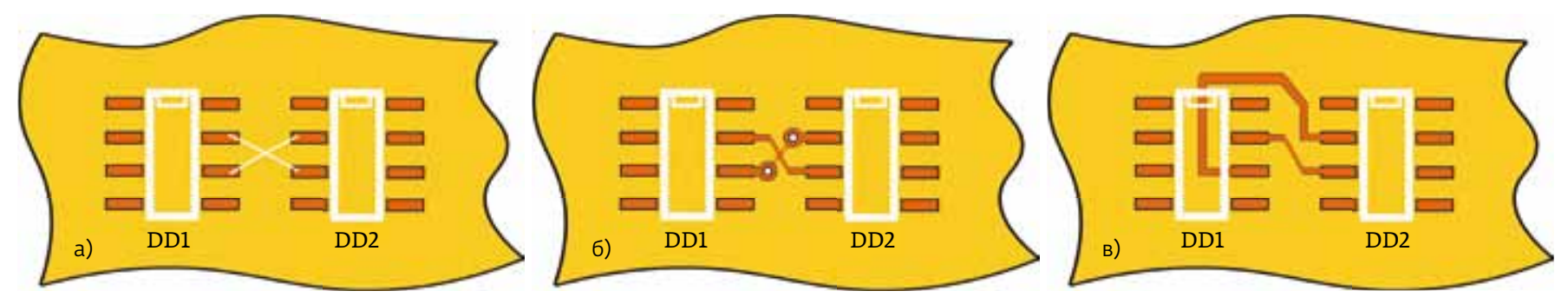

Рис. 5. Пример с перекрестными связями 

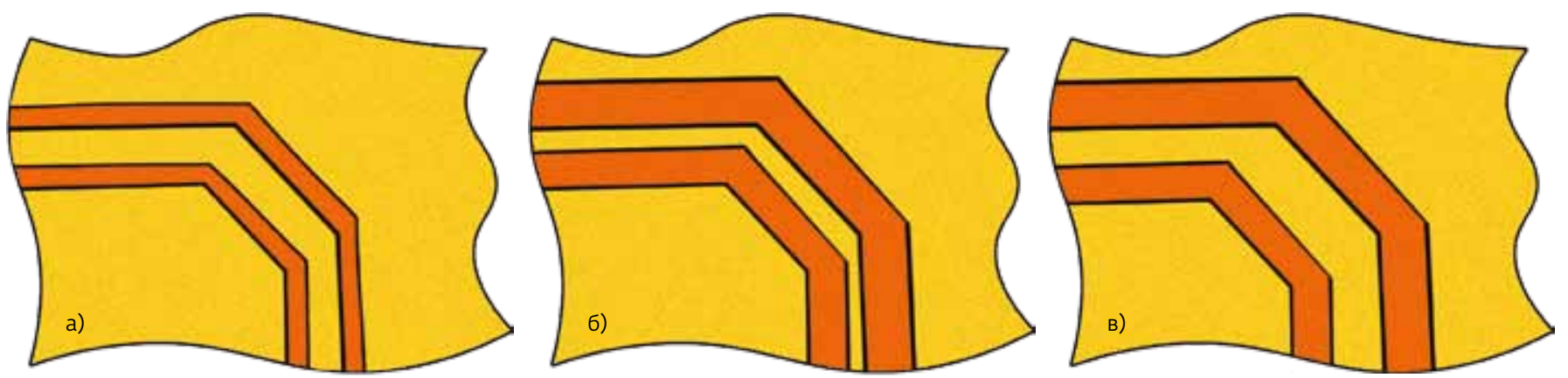

Рис. 6. Варианты увеличения ширины печатных проводников: а - исходная пара: ширина проводника 0,5 мм, расстояние между осевыми линиями 1,27 мм, между внутренними границами 0,77 мм, между внешними границами 1,77 мм; 6 - ширина проводника 1 мм, расстояние между осевыми линиями 1,27 мм, между внутренними границами 0,27 мм, между внешними границами 2,27 мм; в - ширина проводника 1 мм; расстояние между осевыми линиями 1,905 мм, между внутренними границами 0,905 мм, между внешними границами 2,905 мм

зоны контактных площадок проводники можно и нужно расширить. Что касается автотрассировщика, то при установлении правил с минимальной шириной связи он, в отличие от конструктора, приравняет ширину всех проводников к минимальной на протяжении всей длины связей. Конечно, можно воспользоваться локальными правилами с помощью инструментов отдельных настраиваемых зон, таких как Rooms в САПр Altium Designer, но этот механизм требует настройки правил для каждой зоны или группы зон, что является трудоемкой задачей, требующей от конструктора большой сосредоточенности и отнимающей у него много времени

Не нуждается в объяснении тот факт, что при сохранении длины проводника и увеличении его ширины общая площадь, занимаемая токопроводящей структурой, также увеличивается, что чаще всего приводит к необходимости увеличения габаритов печатной платы - еще одна взаимозависимость между параметрами, так или иначе влияющими на надежность. Но есть и менее очевидные эффекты, в которых проявляется такая зависимость.

На рис. 6 показаны два проводника, ширину которых мы намерены увеличить. Возможны два варианта реализации этого намерения. Первый заключается в сокращении расстояния между проводниками (рис. 6б), что ведет к повышению класса точности платы и всем связанным с этим технологическим проблемам, в частности, к увеличению вероятности недотрава с риском короткого замыкания. По второму варианту (рис. 6в) расстояние между проводниками сохраняется, но длина внешнего увеличивается. Выбор варианта может быть осуществлен только конструктором, вне зависимости от того, трассирует ли он плату вручную или пользуется средствами САПР.
Суммируя, можно сказать, что увеличение ширины проводников, как правило, дает существенный положительный результат, однако следует помнить о приведенных выше зависимостях, чтобы предусмотреть и исключить сопутствующие нежелательные эффекты.

\section{КЛАСС ТОЧНОСТИ ПЕЧАТНОЙ ПЛАТЫ}

Когда мы говорим о классе точности ПП, мы имеем в виду комплекс из нескольких параметров - как тех, которые уже обсуждены выше, так и других, также значимых для оценки надежности ПП. Это и расстояния между компонентами, и минимальная ширина проводников и зазоров между ними, а также между проводниками и контактными площадками, и ширина минимальных гарантированных поясков [8]. С одной стороны, повышение класса точности может способствовать улучшению других показателей надежности, в частности, снижению количества переходных отверстий и уменьшению длин связей. С другой стороны, уменьшение минимальной ширины проводника, минимального зазора или же минимального

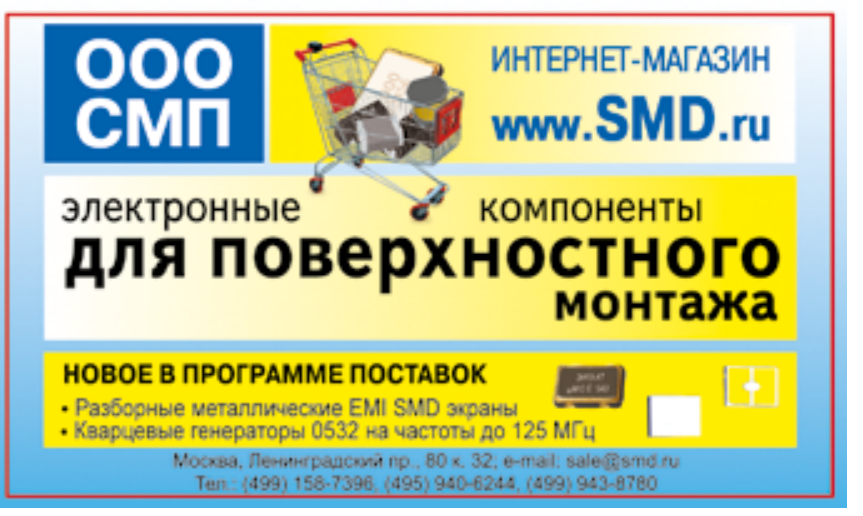


размера гарантированного пояска делает плату более чувствительной к точности поддержания параметров технологических процессов, повышая тем самым риск возникновения описанных выше производственных дефектов.

Таким образом, подтверждается важность учета взаимовлияния различных характеристик ПП: сказать с уверенностью, что увеличение класса точности ведет к улучшению или к ухудшению надежности платы, можно только после того, как будет проведен комплексный анализ всех параметров, имеющих отношение к этому показателю.

\section{ПЛОЩАДЬ ПЕЧАТНОЙ ПЛАТЫ}

Из всех характеристик ПП, в формировании которых играет роль ее площадь, для данной статьи важна лишь устойчивость к механическим воздействиям, так как только этот параметр оказывает прямое влияние на надежность печатной платы: чем больше размер платы, тем более она чувствительна к механическим колебаниям. Наиболее простым выходом в этой ситуации является увеличение числа точек крепления, однако последние занимают довольно большую площадь на поверхности ПП, сравнимую или даже превышающую площадь некоторых микросхем, а также расширяют зону запрета трассировки, увеличивая размер "мертвых зон" на всех слоях печатной платы. В результате этот простой способ обеспечения безопасной эксплуатации крупных ПП в условиях вибраций, ударов и т.п. приводит к очень серьезным последствиям: из-за значительной потери полезной площади конструктор может быть вынужден повысить класс точности ПП либо увеличить количество слоев. Кроме того, существует опасность не разместить на плате все необходимые компоненты; наконец, введение дополнительных точек крепления может быть просто невозможно из-за внешних ограничений - обычно ими бывают требования, обусловленные конструкцией корпуса блока, в который устанавливается ПП.

Получатся, что в описанной ситуации влияние единственного и, казалось бы, несложного параметра площади платы - на все ее характеристики, в том числе на все прочие параметры надежности, настолько велико, что перерастает в перспективу полной переработки проекта, вплоть до размещения электрической схемы устройства не на одной, а на двух платах.

\section{ЗАКЛЮЧЕНИЕ}

Печатная плата - это сложный технологический продукт, и изменения, вносимые в одни элементы ее конструкции, различным образом сказываются на других элементах. Рассмотрение отдельного параметра в отрыве от других может привести к неправильному прогнозированию качества изделия вообще и его надежности в частности. Необходимо не только изучить характер влияния каждого из параметров на надежность ПП, но и, что принципиально важно, определить и формализовать взаимосвязи между ними. На этой основе должна быть построена система весовых коэффициентов к каждому из параметров, которые теперь становятся критериями оценки надежности, и далее - алгоритм, способный быстро и достоверно определять надежность печатных плат.

Сегодня программа, реализующая такой алгоритм, поможет конструктору, позволив оперативно оценивать результат работы автотрассировщика, а также его собственные действия в ходе разработки печатной платы. В перспективе же, с появлением систем проектирования на основе искусственного интеллекта, она станет их элементом - "обратной связью", необходимой для организации процесса обучения нейронной сети.

\section{ЛИТЕРАТУРА}

1. Елшин Ю.М. ГРИФ-4. Информационнопрограммный комплекс расширения функционала САПР Р-CAD200x. Монография [Книга]. М.: ПАО «НПО "Алмаз", 2017. 495 C.

2. Vantsov S., Vasil'ev F., Medvedev A., Khomutskaya O. Quasi-Determinate Model of Thermal Phenomena in Drilling Laminates // Russian Engineering Research. Dec. 2018. Vol. 38. 1074-1076.

3. Ванцов С., Медведев А., Маунг Маунг 3., Хомутская О. Надежность процесса сверления печатных плат, понятие отказа // ЭЛЕКТРОНИКА: Наука, Технология, Бизнес. 2016. № 8. С. 168-172.

4. Ванцов С. В., Васильев Ф. В., Медведев А. М., Хомутская О.В. Влияние нефункциональных контактных площадок на характеристики печатных плат // СТИН. 2019. № 12. С. 31-34.

5. Куликов Н.С., Хомутская О.В., Ванцов С. В. Цифровой метод автоматизированной оценки деформации печатной платы // ЭЛЕКТРОНИКА: Наука, Технология, Бизнес. 2018. № 2. С. 186-191.

6. Можаров В.А., Хомутская О.В. Влияние нефункциональных контактных площадок на различные характеристики печатных плат // ЭЛЕКТРОНИКА: Наука, Технология, Бизнес. 2017. № 2. С. 166-176.

7. Васильев Ф.В., Медведев А. М., Сергеев А. В. Токонесущая способность печатных проводников // Практическая силовая электроника. 2015. № 4. C. $52-55$.

8. ГОСТ Р 53429-2009. Платы печатные. Основные параметры конструкции. - М.: Стандартинформ, 2010. 11 c. 


\section{害ТЕСТПРИБОР}

\section{Высококачественные покрытия $\mathrm{Ni}, \mathrm{Au}$ произво Аства АО «ТЕСТПРИБОР»}

Различные метамииеские покрытия, выполненные электрохимическим, химическим и Аругими способами находят активное применение при проивводстве широкого спектра изАелий микроэлектроники.
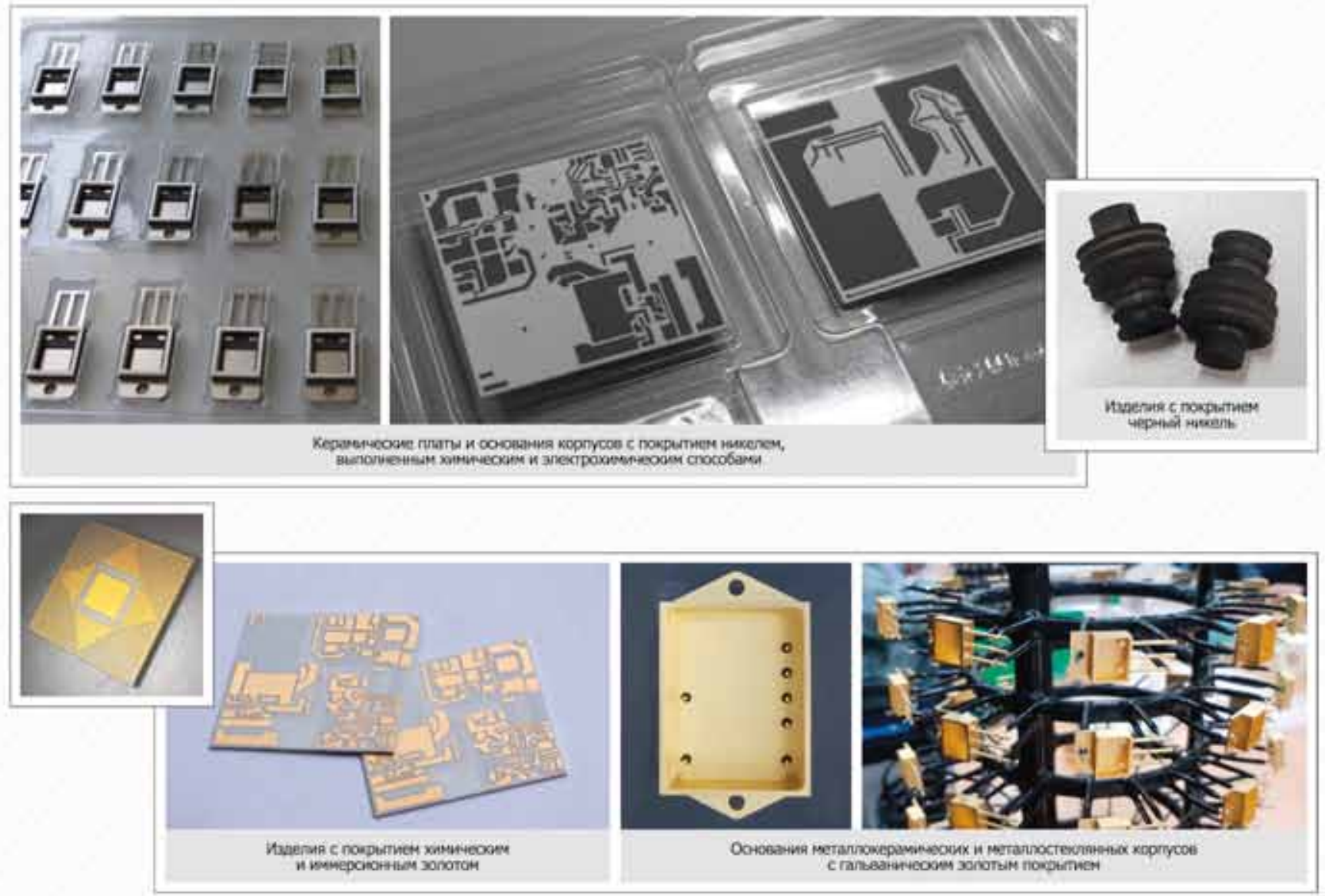

Производство компании «ТЕСТПРИБОР» в рамках освоения разиичного рода экспериментальной и серийной продукции владеет следующими технологиями нанесения покрытий:

- электрохимическое никелирование из растворов различных составов: толщина слоя покрытия до 30 мкм;

- электрохимическое никелирование сплавом никель-фосфор: толщина слоя покрытия до 30 мкм;

- химическое никелирование сплавами никель-фосфор, никель-бор; гарантированная толщина покрытия до $10 \mathrm{MKM}$
" электрохимическое золочение: толщина покрытия до 10 мкM

- химическое и иммерсионное золочение - толщина покрытия, нанесенного химическим способом, от 0,5 Ао 2,5 мкм, толщина слоя иммерсионного покрытия до 0,15 мкм

- «черный никель» 
Важным фактором успеха при выполнении покрытия электрохимическим методом является грамотно спроектированная оснастка, позволяющая:

- наносить покрытие на большое количество изделий с разницей в толщине от изделия к изделию не превышающей 1-2\% внутри партии и не 6олее $5 \%$ по поверхности одного изделия в зависимости от его формы и особенностей топологии;

- наносить покрытие разной толщины на изолированные друг от друга части изделия, что особенно важно в случае нанесения золотого покрытия и позволяет снизить себестоимость детали за счет снижения расхода золота на покрытие тех участков, которые удаляются в процессе монтажа корпуса в прибор при сборке конечного устройства, либо выполнить частичное покрытие только технологически важных участков;

- минимизировать размер паразитных точек контакта, либо устранять их методом локального покрытия;

- использовать химически стойкие материалы (нержавеющая сталь, полиэтилен, поливинилхлорид) для снижения рисков загрязнения растворов продуктами травления подвески.

\section{ПРИМЕРЫ ОРИГИНАЛЬНОЙ ОСНАСТКИ ДЛЯ ГАЛЬВАНИЧЕСКОГО ПОКРЫТИЯ, СПРОЕКТИРОВАННОЙ И ИЗГОТОВЛЕННОЙ АО «ТЕСТПРИБОР»:}

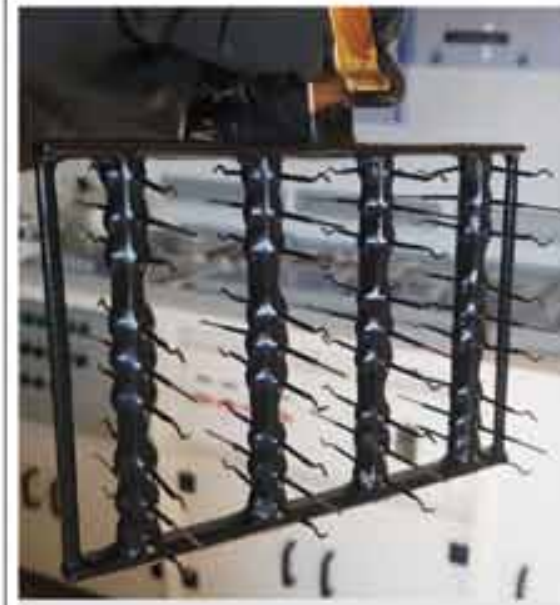

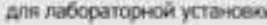

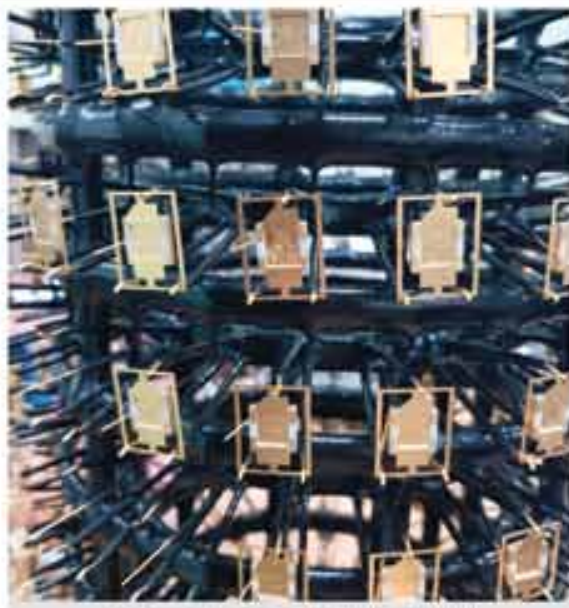

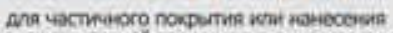

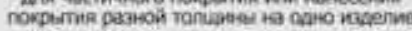

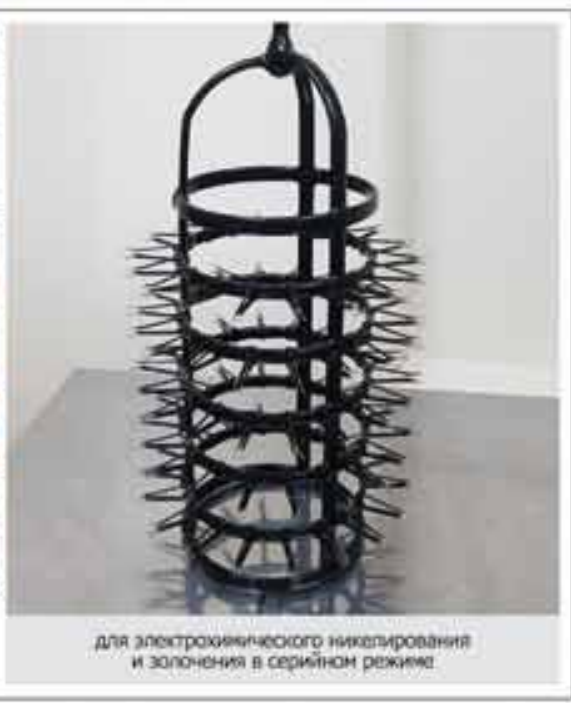

Высокое качество гальванических покрытий,

выполненных на производстве АО «ТЕСТПРИБОР», о6еспечено:

- специально спроектированным оборудованием и оснасткой, позволяіщим получать повторяемость по толщине и характеристикам покрытий внутри партии и достигать равномерности покрытия на одном изделии настолько, насколько позволяют его геометрические характеристики.

- качеством используемых растворов и постоянством технологических параметров, под четким контролем производственной лаборатории и инженерного персонала.
- грамотно подобранными составами, позволяющими наносить как классические покрытия, так и редко используемые при обычной практике - например, золотое покрытие, выполненное химическим способом до толщины более 2 мкм, что способствует осуществлению широкого спектра задач поставленных заказчиками компании.

- контролем качества изделий, производимым в несколько этапов по многим параметрам на $100 \%$ продукции, что снижает вероятность попадания бракованных изделий к заказчику. 